\title{
Macrophages enter CAR immunotherapy
}

\author{
$\mathrm{CAR}^{+}$macrophages modulate the tumor milieu and lead to antitumor \\ immune responses.
}

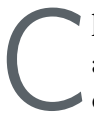
himeric antigen receptors (CARs) are engineered molecules that combine the specificity of antibodies with the downstream signaling of T cells. Expression of CARs on T cells has shown enormous promise in the treatment of malignancies. However, CAR-T cell therapy is often hampered by the inability of $\mathrm{T}$ cells to penetrate solid tumors, as well as the inhibitory tumor microenvironment (TME).

To overcome this challenge, Michael Klichinsky in the laboratory of Saar Gill at the University of Pennsylvania and colleagues have transduced CARs into macrophages (CAR-Ms) to show that CAR-Ms could efficiently contribute to the antitumor response. "We decided to take a step back and see if there is an immune cell type better suited for solid tumors," explains Klichinsky, the first author of the report. Macrophages form an indispensable part of immune response, performing a host of tasks crucial for the regulation of the immune system. Not only are they professional antigen-presenting cells, but macrophages also actively participate in the immune response through phagocytosis and clearance of cellular debris.

To first test the specific phagocytosis of tumor cells in vitro, CAR-Ms were expressed on THP-1 macrophage cell lines and cocultured with antigen-positive tumor cells to demonstrate the specific clearance of targets. Getting CARs to express in these cells, however, proved to be a challenge because of the non-replicative nature of macrophages. "We spent a lot of time testing suitable vectors to transduce primary human macrophages with high efficiency and viability", says Klichinsky. Finally, a modified replication-incompetent adenovirus was found to induce sustained expression of CARs on donor-derived macrophages. In vitro coculture of anti-HER2 CAR-Ms with SKOV3 cells (an ovarian cancer cell line) showed that CAR-Ms mediated a time- and dose-dependent eradication of tumor cells.

Having established the antitumor activity of CAR-Ms in vitro, the authors tested their effects in vivo on mice modified to lack T cells, B cells and natural killer cells. These NSGS mice allow researchers to establish a growing human tumor without triggering a host immune response. Mice bearing SKOV3 lung or peritoneal metastases were treated with anti-HER2 CAR-Ms and showed a distinct decrease in tumor volume as well as an increased overall survival compared to control mice.

One major concern in macrophage-based therapies is the high plasticity of macrophages. In response to external stimuli like cytokines, macrophages differentiate into antitumor proinflammatory M1 or immunosuppressive M2 phenotypes. Using RNA-seq, the team showed that gene expression in CAR-Ms closely matched that of M1 macrophages, leading the researchers to investigate whether CAR-Ms could influence the phenotype of bystander macrophages. When SKOV 3 cells and CAR-Ms were transferred into humanized mice, single-cell RNA-seq of the TME revealed that CAR-Ms induced a proinflammatory milieu, skewing the bystander myeloid cells towards a M1 phenotype. Remarkably, CAR-Ms also induced positive effects on other cell types by activating immature dendritic cells and recruiting activated CD8+ $\mathrm{T}$ cells to the tumor site, thus enhancing the overall antitumor response.

"Our next step will be to look at rationally selective combination therapy. We will select treatments like radiation or immune-checkpoint therapy that may have a synergistic effect in combination with CAR macrophages," notes Klichinsky. The team, now at Carisma Therapeutics, is also poised to test anti-HER2 CAR-Ms in a first-in-human clinical trial.

Harnessing the power of innate immune cells, these results present a novel tool for treatment of solid tumors and open up the possibility of the development of new and exciting immunotherapeutic modalities.

Madhura Mukhopadhyay

Published online: 4 June 2020

https://doi.org/10.1038/s41592-020-0862-4

Research paper

Klichinsky, M. et al. Human chimeric antigen receptor macrophages for cancer immunotherapy. Nat Biotechnol. https://doi.org/10.1038/s41587-020-0462-y (2020)

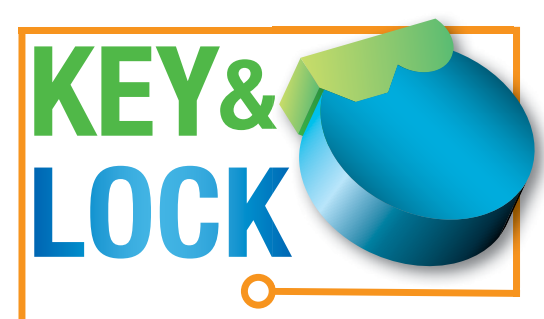

\section{Working together we open the potential of scientific innovation}

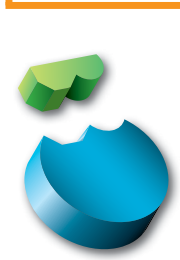

Our enzymes have been discovering facts about life for decades.

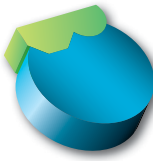

That's why Worthington, the primary enzyme

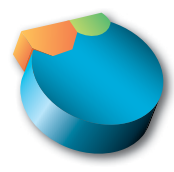
producer, has more citations in respected journals like Nature Methods and rank higher in search

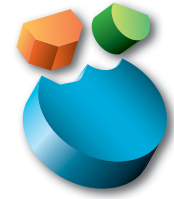
engines like $B i o z^{\circledR}$.

COLLAGENASES $\rightarrow 99 \%$ Bioz Rating
DNASE I $\longrightarrow 99 \%$ Bioz Rating
PAPAIN $\longrightarrow 99 \%$ Bioz Rating

Unlock the power of Worthington with our new Introduction to

Enzymes guide. Go to: Worthington-Biochem.com

\section{INTRODUCTION TO ENZYMES}

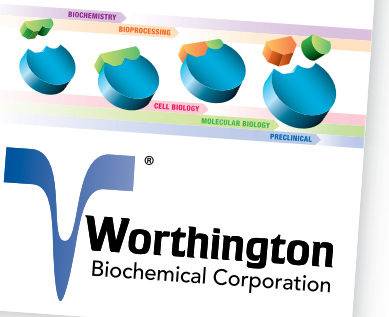

